\title{
Hypertension due to a deoxycorticosterone- secreting adrenal tumour diagnosed during pregnancy
}

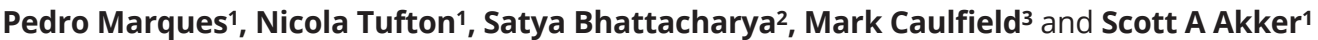 \\ 'Department of Endocrinology, St. Bartholomew's Hospital, West Smithfield, London, UK, 2Hepatobiliary and \\ Pancreatic Surgery Unit, The Royal London Hospital, London, UK, and ${ }^{3}$ Clinical Pharmacology, William Harvey \\ Research Institute, Queen Mary University of London, London, UK
}

Correspondence should be addressed to P Marques Email

pedro.miguel.sousa. marques@gmail.com

\section{Summary}

Mineralocorticoid hypertension is most often caused by autonomous overproduction of aldosterone, but excess of other mineralocorticoid precursors can lead to a similar presentation. 11-Deoxycorticosterone (DOC) excess, which can occur in 11- $\beta$ hydroxylase or 17- $\alpha$ hydroxylase deficiencies, in DOC-producing adrenocortical tumours or in patients taking 11- $\beta$ hydroxylase inhibitors, may cause mineralocorticoid hypertension. We report a 35-year-old woman who in the third trimester of pregnancy was found to have a large adrenal mass on routine obstetric ultrasound. On referral to our unit, persistent hypertension and long-standing hypokalaemia was noted, despite good compliance with multiple antihypertensives. Ten years earlier, she had hypertension noted in pregnancy which had persisted after delivery. A MRI scan confirmed the presence of a $12 \mathrm{~cm}$ adrenal mass and biochemistry revealed high levels of DOC and low/ normal renin, aldosterone and dehydroepiandrosterone, with normal catecholamine levels. The patient was treated with antihypertensives until obstetric delivery, following which she underwent an adrenalectomy. Histology confirmed a large adrenal cortical neoplasm of uncertain malignant potential. Postoperatively, blood pressure and serum potassium normalised, and the antihypertensive medication was stopped. Over 10 years of follow-up, she remains asymptomatic with normal DOC measurements. This case should alert clinicians to the possibility of a diagnosis of a DOC-producing adrenal tumours in patients with adrenal nodules and apparent mineralocorticoid hypertension in the presence of low or normal levels of aldosterone. The associated diagnostic and management challenges are discussed.

\section{Learning points:}

- Hypermineralocorticoidism is characterised by hypertension, volume expansion and hypokalaemic alkalosis and is most commonly due to overproduction of aldosterone. However, excess of other mineralocorticoid products, such as DOC, lead to the same syndrome but with normal or low aldosterone levels.

- The differential diagnosis of resistant hypertension with low renin and low/normal aldosterone includes congenital adrenal hyperplasia, syndrome of apparent mineralocorticoid excess, Cushing's syndrome, Liddle's syndrome and 11-deoxycorticosterone-producing tumours.

- DOC is one intermediate product in the mineralocorticoid synthesis with weaker activity than aldosterone. However, marked DOC excess seen in 11- $\beta$ hydroxylase or 17- $\alpha$ hydroxylase deficiencies in DOC-producing adrenocortical tumours or in patients taking 11- $\beta$ hydroxylase inhibitors, may cause mineralocorticoid hypertension.

- Excessive production of DOC in adrenocortical tumours has been attributed to reduced activity of the enzymes 11- $\beta$ hydroxylase and 17- $\alpha$ hydroxylase and increased activity of $21-\alpha$ hydroxylase.

- The diagnosis of DOC-producing adrenal tumours is challenging because of its rarity and poor availability of DOC laboratory assays. 


\section{Background}

Mineralocorticoid-induced hypertension is common and normally associated with autonomous aldosterone overproduction (primary hyperaldosteronism). However, other mineralocorticoid hormones such 11-deoxycorticosterone (DOC) may be responsible for a similar condition $(1,2)$. The rarity of DOC-producing adrenocortical tumours (3) and poor availability of laboratory assays to measure mineralocorticoid products other than aldosterone cause diagnostic challenges. This case highlights the diagnostic challenges, with the patient's diagnosis remaining hidden for at least 10 years.

\section{Case presentation}

A 35-year-old female was referred for evaluation of an adrenal mass detected by routine ultrasound performed during the third trimester of her seventh pregnancy. Ten years previously, she had been diagnosed with hypertension which had been managed with antihypertensives. In her fifth pregnancy, she developed pre-eclampsia resulting in a premature emergency caesarean section. She also reported three past miscarriages for unknown reasons. She denied weight changes before pregnancy and reported a regular menstrual cycle. She reported no hirsutism, acne, headaches, palpitations, oedema or other symptoms suggestive of Cushing's syndrome. Her family history was unremarkable for hypertension, and there was no familial consanguinity history. On presentation, her blood pressure was $140 / 80 \mathrm{mmHg}$ on labetalol $200 \mathrm{mg}$ (three times a day), doxazosin $8 \mathrm{mg}$ (once daily) and diltiazem $200 \mathrm{mg}$ (twice daily). She was taking oral potassium supplements due to long-standing hypokalaemia and denied any exogenous glucocorticoid administration or chronic ingestion of liquorice. Physical examination revealed a gravid uterus but was otherwise unremarkable, with no evidence of any endocrinopathy.

\section{Investigation}

The serum potassium was $3.7 \mathrm{mmol} / \mathrm{L}$ on supplements. Two twenty four-hour urinary catecholamine collections were normal. The renin and aldosterone were both low, with a morning serum cortisol of $390 \mathrm{nmol} / \mathrm{L}$ and an ACTH level of $44 \mathrm{ng} / \mathrm{dL}$ (Table 1). Considering the hypertension in the context of low renin and aldosterone and history of chronic hypokalaemia, it was suspected that other mineralocorticoid hormones could be elevated.
A non-contrast MRI scan undertaken during pregnancy revealed a $12.1 \times 10.5 \times 9.0 \mathrm{~cm}$ left-sided adrenal mass, which was complex and cystic in nature (Fig. 1). A postpartum abdominal CT scan demonstrated a large heterogeneous mass thought to be arising from the left adrenal and indenting the tail of the pancreas (Fig. 1).

The 11-DOC result, sent postpartum, was markedly elevated at $9741 \mathrm{pmol} / \mathrm{L}$ (reference range: 121-514) with normal corticosterone levels. Table 1 summarises postpartum biochemistry results.

\section{Treatment}

Surgical resection was planned for after delivery, as the diagnosis of an adrenal tumour was made during the third trimester of pregnancy, with evidence of this being a longstanding condition (i.e. duration of hypertension and hypokalaemia). The hypertension was controlled with labetalol, doxazosin and diltiazem. Mineralocorticoid antagonists were not used as these are contraindicated in pregnancy. The patient delivered a healthy boy at 39 weeks gestational age by caesarean section, with no complications.

Surgical resection of the left adrenal tumour took place 3 months postpartum. The histology was compatible with an adrenocortical neoplasm of uncertain malignant potential. The tumour was $11 \times 7.5 \times 6.0 \mathrm{~cm}$ and composed of two cell types: cells with abundant clear cytoplasm and nuclei with small nucleoli and cells with eosinophilic

Table 1 Summary of patient's biochemistry results, postpartum and prior to adrenal surgery, showing high suppressed renin and aldosterone and markedly raised levels of 11-DOC.

\begin{tabular}{|c|c|c|}
\hline Laboratory test & Result & Reference range/units \\
\hline Serum aldosterone & 224 & $330-830 \mathrm{pmol} / \mathrm{L}$ \\
\hline Serum renin activity & 0.01 & $0.15-2.1 \mathrm{pmol} / \mathrm{ml} / \mathrm{h}$ \\
\hline Corticosterone & 17.35 & $5.78-23.12 \mathrm{nmol} / \mathrm{L}$ \\
\hline 11-Deoxycorticosterone & 9741 & $121-514 \mathrm{pmol} / \mathrm{L}$ \\
\hline Plasma adrenaline & $<4$ & $<4 \mathrm{nmol} / \mathrm{L}$ \\
\hline Plasma noradrenaline & 2.60 & $0-4.14 \mathrm{nmol} / \mathrm{L}$ \\
\hline Serum cortisol (9: 00h) & 390 & $\mathrm{nmol} / \mathrm{L}$ \\
\hline ACTH & 44 & $\mathrm{ng} / \mathrm{dL}$ \\
\hline Dehydroepiandrosterone & 0.9 & $1.9-9.4 \mu \mathrm{mol} / \mathrm{L}$ \\
\hline Androstenedione & 2.1 & $3-8 \mu \mathrm{mol} / \mathrm{L}$ \\
\hline Testosterone & $<0.7$ & $0-2.9 \mathrm{nmol} / \mathrm{L}$ \\
\hline Oestradiol & 466 & $\mathrm{pmol} / \mathrm{L}$ \\
\hline $\begin{array}{l}\text { Sex hormone-binding } \\
\text { globulin }\end{array}$ & 60 & $22-126 \mathrm{nmol} / \mathrm{L}$ \\
\hline TSH & 0.7 & $0.35-5.5 \mathrm{mUI} / \mathrm{L}$ \\
\hline Free T4 & 17.3 & $10.5-20 \mathrm{pmol} / \mathrm{L}$ \\
\hline $\mathrm{HbA} 1 \mathrm{C}$ & 5.6 & $\%$ \\
\hline
\end{tabular}



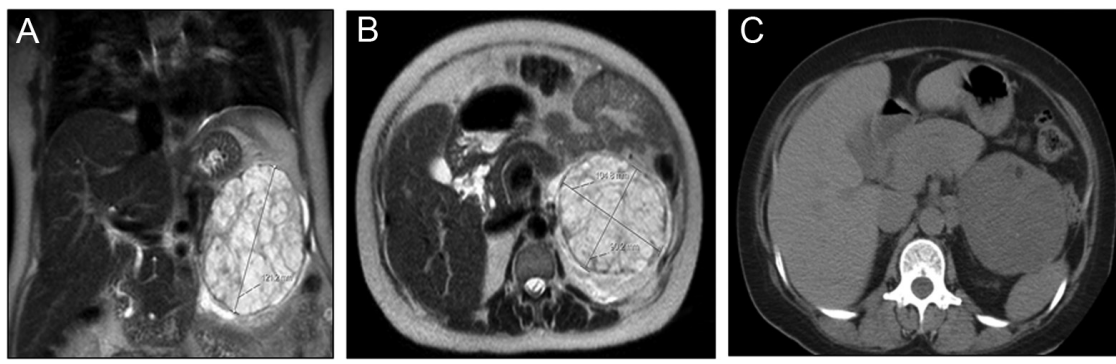

Figure 1

Non-contrast abdominal MRI scan undertaken during the third gestational trimester (A and $\mathrm{B}$ ) and postpartum abdominal CT scan (C) showing a cystic and heterogeneous $12.1 \times 10.5 \times 9.0 \mathrm{~cm}$ left adrenal tumour.

cytoplasm having vesicular nuclei with prominent nucleoli. There was mild nuclear pleomorphism, but no mitotic figures, and areas of myxoid change and haemorrhage, but no necrosis. Capsular or vascular invasion were not observed, and the Ki-67 index was less than $1 \%$ (Weiss score 2).

\section{Outcome and follow-up}

Postoperatively, blood pressure and serum potassium normalised off medications. Over 10 years of follow-up, the patient has remained asymptomatic with normal blood pressure and potassium levels, requiring no medication or supplements. Measurements of plasma DOC levels have remained within the normal reference range, and postoperative imaging during the first 4 years of follow-up excluded either locoregional recurrence or systemic disease.

\section{Discussion}

Autonomous excess production of mineralocorticoid hormones causes hypertension, volume expansion, hypokalaemia and renin suppression - the hallmarks of hypermineralocorticoidism (1). Mineralocorticoid hypertension is one of the leading causes of secondary hypertension and is usually due to autonomous overproduction of aldosterone, that is, primary hyperaldosteronism (1). However, excess of other mineralocorticoid products such as DOC, 18-hydroxy11-DOC, 19-Nor-DOC, 19-hydroxy-DOC and 11-deoxycortisol may induce a similar condition but with a low/suppressed aldosterone $(2,4)$. In this setting, aldosterone is normally low because the alternative mineralocorticoid excess increases intravascular volume and suppresses the renin-angiotensin system (1).

The differential diagnosis of hypertension with low renin and aldosterone includes congenital adrenal hyperplasia (CAH), syndrome of apparent mineralocorticoid excess, Cushing's syndrome, Liddle's syndrome and DOC-producing adrenal tumours $(1,5)$.

In patients with resistant hypertension, hypokalaemia and accompanied by low/normal plasma renin activity/ levels and low/normal serum aldosterone levels, Cushing's syndrome is an important diagnosis to be considered and ruled out $(1,5,6)$. Cushing's syndrome associated with this phenotype is usually clinically apparent as the principal mechanism is thought to be due to very high cortisol levels saturating renal 11 $\beta$-hydroxysteroid dehydrogenase type 2 , thereby preventing the inactivation of cortisol to

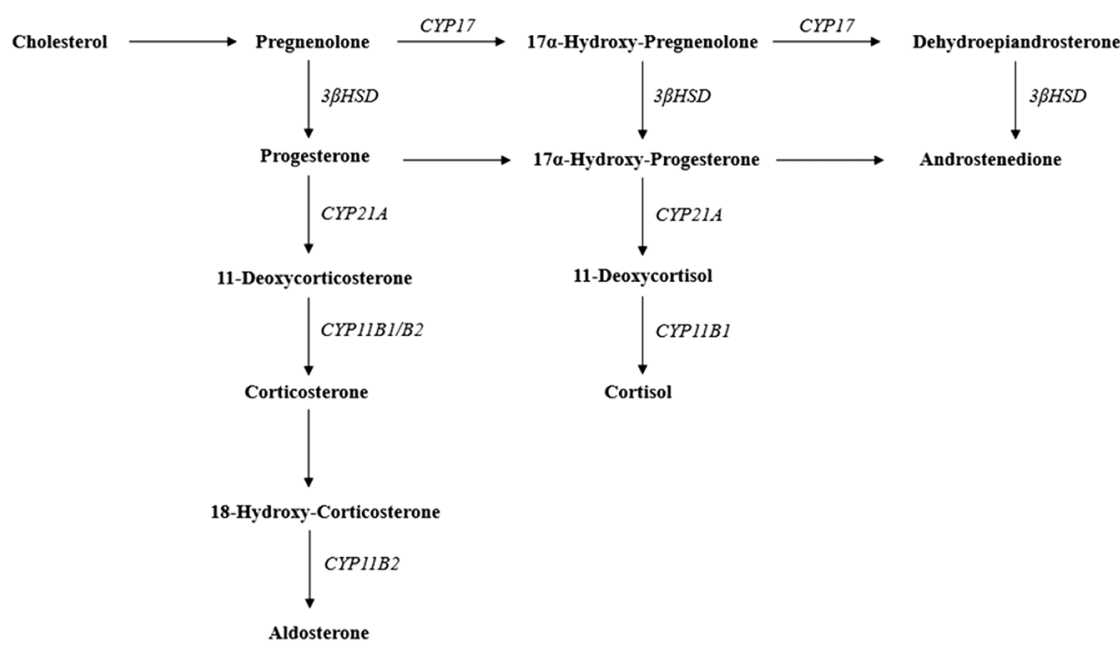

Figure 2

The adrenal steroidogenesis. The adrenal steroidogenesis is controlled by cytochrome P450 enzymes: CYP21A encodes $21 \alpha$ hydroxylase; CYP11B1 encodes $11 \beta$ hydroxylase; CYP11B2 encodes aldosterone synthase; CYP17 encodes $17 \alpha$ hydroxylase. The $3 \beta H S D$ (3 $\beta$-hydroxysteroid dehydrogenase) activity is mediated by a single non-P450 microsomal enzyme (1). 
cortisone and allowing binding to the mineralocorticoid receptor $(6,7)$ and/or due to elevated angiotensinogen levels in such patients (6).

CAH due to 11- $\beta$ hydroxylase deficiency also presents with resistant hypertension associated with increased DOC levels, low aldosterone, hypokalaemia and eucortisolaemia. However it normally presents very early in life and is accompanied with significant virilisation or hirsutism of the infant. As a recessive condition it is often associated with familial consanguinity. $\mathrm{CAH}$ is more frequent than DOC-producing tumours, but rarely has a late presentation in adulthood $(4,5)$.

Syndrome of apparent mineralocorticoid excess is a rare autosomal recessive disorder due to mutations in the gene encoding the 11ß-hydroxysteroid dehydrogenase type 2 enzyme (which converts cortisol to inactive cortisone in the renal tubules) resulting in its deficiency, causing therefore sodium retention, potassium wasting and severe hypokalaemic hypertension (8). This syndrome is commonly found in patients with resistant hypertension, extensive target organ damage, nephrocalcinosis and renal failure and with familial consanguinity history. A similar clinical scenario occurs following the ingestion of bioflavonoids, liquorice and carbenoxolone, which are competitive inhibitors of the $11 \beta$-hydroxysteroid dehydrogenase type 2 enzyme $(1,5,8,9)$.

Liddle's syndrome is a condition associated with activating mutations of $\beta$ and $\gamma$ subunits of the renal epithelial sodium channel (leading to sodium reabsorption and subsequently to hypermineralocorticoidism manifestations such as hypertension, hypokalaemia and metabolic acidosis) and should be considered when the above entities are excluded (5).

DOC is an intermediate product in mineralocorticoid synthesis (Fig. 2), produced in the zona fasciculata and displays a weak mineralocorticoid activity in comparison to aldosterone (approximately 1/20) (4). DOC excess can be found in patients with 11- $\beta$ hydroxylase or $17-\alpha$ hydroxylase deficiencies, DOC-producing adrenocortical tumours or in patients taking 11- $\beta$ hydroxylase inhibitors (e.g. metyrapone or mitotane) $(1,3,10)$. As DOC does not have a potent mineralocorticoid action, high levels are necessary to induce hypertension and hypokalaemia $(2,3)$. In this case there was a remarkable DOC elevation, approximately 19 times above the upper limit of the reference range, but other reported cases were found with even higher DOC elevations $(3,9)$.

In DOC-producing adrenal tumours, co-secretion of corticosterone may occur, as well as other hormones such as androgens, oestrogens or cortisol $(3,10)$. Adrenal tumours with mixed steroid-secreting pattern should be evaluated for malignancy (10). Adrenocortical carcinomas are often associated with elevated mineralocorticoid precursors, including DOC, whereas benign adrenal tumours usually have normal or low mineralocorticoid precursors (10). The excessive DOC production in adrenocortical tumours has been attributed to reduced activity of 11- $\beta$ hydroxylase and 17- $\alpha$ hydroxylase (Fig. 2) (9), as supported by the demonstration of low or absent activity of cytochrome $\mathrm{P}_{450}{ }_{11 \beta}$ and $\mathrm{P}_{450_{17 \alpha}}$ in DOC-producing adrenal adenomas $(2,9,11)$ and carcinomas $(12)$. DOC-producing adrenal adenomas have lower DOC content than the normal adrenal cortex despite the increased production, probably because DOC is actively released in circulation (2).

The diagnosis of DOC-producing adrenal tumours is challenging due to its rarity and DOC assays have limited availability. This condition can be misdiagnosed as a non-functioning adenoma if DOC is not assessed (10). Moreover, DOC-producing adrenal tumours may occur concomitantly with primary hyperaldosteronism masking the key feature of DOC-producing tumours (suppressed aldosterone, alongside with suppressed renin), raising additional diagnostic challenges $(2,10)$.

Here, we present the first case formally diagnosed during pregnancy, although it is likely that the patient had been suffering from this condition for at least 10 years when she was first diagnosed with hypokalaemic hypertension. This case aims to alert clinicians to the existence of DOC-producing adrenal tumours, which should be considered in adult patients with adrenal tumours and apparent mineralocorticoid resistant hypertension with low aldosterone and renin levels.

\section{Declaration of interest}

The authors declare that there is no conflict of interest that could be perceived as prejudicing the impartiality of the research reported.

\section{Funding}

P M is supported by Barts and The London Charity Clinical Research Training Fellowship. N T is supported by The Medical College of Saint Bartholomew's Hospital Trust training fellowship.

\section{Patient consent}

Informed consent was obtained from the patient.

\section{Author contribution statement}

$\mathrm{P} \mathrm{M}$ and $\mathrm{N} T$ were responsible for data collection, presentation and for writing the manuscript. M C, S B and S A A were the senior clinicians overseeing the care of the patient and provided significant input in terms of literature research, critical discussion and revision of the manuscript. 


\section{References}

1 Biglieri EG \& Kater CE. Steroid characteristics of mineralocorticoid adrenocortical hypertension. Clinical Chemistry 199137 1843-1848.

2 Toyoda Y, Mizukoshi M, Umemoto M, Kuchii M, Ueyama K, Tomimoto S, Baba A, Shima H, Nishio I \& Masuyama Y. Adrenal tumor producing 11-deoxycorticosterone, 18-hydroxy-11deoxycorticosterone and aldosterone. Internal Medicine 199635 123-128. (https://doi.org/10.2169/internalmedicine.35.123)

3 Irony I, Biglieri EG, Perloff D \& Rubinoff H. Pathophysiology of deoxycorticosterone-secreting adrenal tumors. Journal of Clinical Endocrinology and Metabolism 198765 836-840. (https://doi. org/10.1210/jcem-65-5-836)

4 Oyama K, Nozaki A, Horie I, Shigematsu K, Takehara K, Ando T \& Kawakami A. Deoxycorticosterone-producing adenoma concomitant with aldosterone-producing microadenoma: a challenging combination. Endocrine Practice 201420 e171-e175. (https://doi. org/10.4158/EP14175.CR)

5 Gupta S, Melendez J \& Khanna A. Deoxycorticosterone producing tumor as a cause of resistant hypertension. Case Reports in Medicine 20102010 372719. (https://doi.org/10.1155/2010/372719)

6 van der Pas R, van Esch JH, de Bruin C, Danser AH, Pereira AM, Zelissen PM, Netea-Maier R, Sprij-Mooij DM, van den BergGarrelds IM, van Schaik RH, et al. Cushing's disease and hypertension: in vivo and in vitro study of the role of the reninangiotensin-aldosterone system and effects of medical therapy. European Journal of Endocrinology 2014170 181-191. (https://doi. org/10.1530/EJE-13-0477)
7 Sharma ST \& Nieman LK. Cushing's syndrome: all variants, detection, and treatment. Endocrinology and Metabolism Clinics of North America 201140 379-391, viii-ix. (https://doi.org/10.1016/j. ecl.2011.01.006)

8 Ferrari P. The role of 11beta-hydroxysteroid dehydrogenase type 2 in human hypertension. Biochimica and Biophysica Acta 20101802 1178-1187. (https://doi.org/10.1016/j. bbadis.2009.10.017)

9 Wada N, Kubo M, Kijima H, Yamane Y, Nishikawa T, Sasano H \& Koike T. A case of deoxycorticosterone-producing adrenal adenoma. Endocrine Journal 199542 637-642. (https://doi.org/10.1507/ endocrj.42.637)

10 Aupetit-Faisant B, Blanchouin-Emeric N, Tenenbaum F, Battaglia C, Tabarin A, Amar J, Kuttenn F, Warnet A, Assayag M \& Chamontin B. Plasma levels of aldosterone versus aldosterone precursors: a way to estimate the malignancy of asymptomatic and nonsecretory adrenal tumors: a French Retrospective Multicentric Study. Journal of Clinical Endocrinology and Metabolism 199580 2715-2721. (https://doi. org/10.1210/jcem.80.9.7673414)

11 Shibata H, Suzuki H, Ogishima T, Ishimura Y \& Saruta T. Significance of steroidogenic enzymes in the pathogenesis of adrenal tumour. Acta Endocrinologica 1993128 235-242. (https://doi.org/10.1530/ acta.0.1280235)

12 Kelly WF, O’Hare MJ, Loizou S, Davies D \& Laing I. Hypermineralocorticism without excessive aldosterone secretion: an adrenal carcinoma producing deoxycorticosterone. Clinical Endocrinology 198217 353-361. (https://doi. org/10.1111/j.1365-2265.1982.tb01600.x)

Received in final form 8 March 2019

Accepted 11 April 2019 